\title{
Modular transformation and boundary states in logarithmic conformal field theory
}

\author{
Shinsuke Kawai * and John F. Wheater ${ }^{\dagger}$ \\ Theoretical Physics, Department of Physics, University of Oxford, 1 Keble Road, Oxford OX1 3NP, UK
}

(October 27, 2018)

\begin{abstract}
We study the $c=-2$ model of logarithmic conformal field theory in the presence of a boundary using symplectic fermions. We find boundary states with consistent modular properties. A peculiar feature of this model is that the vacuum representation corresponding to the identity operator is a sub-representation of a "reducible but indecomposable" larger representation. This leads to unusual properties, such as the failure of the Verlinde formula. Despite such complexities in the structure of modules, our results suggest that logarithmic conformal field theories admit bona fide boundary states.

PACS number(s): 11.10.Kk, 11.25.Hf, 68.35.Rh
\end{abstract}

\section{INTRODUCTION}

The existence of critical systems with logarithmic correlation functions has been known for some time. It was almost a decade ago when Saleur [1] pointed out the existence of logarithmic behavior in the correlation functions of some geometrical critical systems. Subsequently, the importance of such logarithmic terms in conformal field theories (CFTs) was emphasized by Gurarie [2], who pointed out that this phenomenon occurs when two (or more) primary operators have scaling dimensions differing by integers, and that these operators are not diagonal under conformal transformations but they form Jordan cells. Such theories had never been studied seriously till then, mainly because they are not unitary. Importantly, however, non-unitary does not mean unrealistic - there are a large number of non-unitary models relevant in statistical and high-energy physics. Such logarithmic conformal field theories (LCFTs) are now known to be quite ubiquitous [3], and are being studied not only for their mathematical interests, but also for their potential applicability in string theory and statistical physics.

Although much work has been done in recent years, our knowledge of LCFTs is still incomplete. In particular, the behavior of such systems in the presence of a boundary is not fully understood. In ordinary CFTs, the system near the boundary is described by bulk and boundary operators. Then, once the physical boundary states are identified, the scaling properties of the given system are completely understood. At least in the case of unitary minimal models, such physically consistent boundary states are most systematically obtained by the "fusion method" of Cardy 沟. This method uses the duality of open-string

*s.kawai1@physics.ox.ac.uk

${ }^{\dagger} \mathrm{j}$.wheater1@physics.ox.ac.uk and closed-string channels; one partition function is calculated in two different ways, and consistency gives nontrivial constraints on the boundary states which are realized physically. In LCFTs, however, the validity of this method, and even the existence of well-defined physical boundary states, has not been clear. The main obstacle is that operators in LCFTs are not classified into simple conformal towers which split the partition function into Virasoro characters [5]. The purpose of this note is to show that Cardy's method is applicable to LCFTs and that LCFTs allow physically consistent boundary states.

In this paper we focus on the triplet model of $c=-2$ which has explicit Lagrangian representation [1,6]:

$$
S=\frac{1}{\pi} \int d^{2} z(\eta \bar{\partial} \xi+\bar{\eta} \partial \bar{\xi}),
$$

where $\eta$ and $\xi$ are fermionic fields of scaling dimensions 1 and 0 , respectively, with operator products $\eta(z) \xi(w) \sim$ $\xi(z) \eta(w) \sim 1 /(z-w)$. This is nothing but the fermionic ghost system with central charge -2 . Our strategy is to derive explicit boundary states in Fock space and then to study their modular properties. Before discussing boundary states, we shall briefly summarize how the bulk chiral theory is constructed from the action (11) and thereby define our model precisely [6,7]. The energy-momentum tensor of this system is $T(z)=: \partial \xi(z) \eta(z)$, from which the central charge is read off as $c=-2$. The system has a $U(1)$ symmetry whose current is $J_{U(1)}(z)=: \xi(z) \eta(z)$ :. Due to this symmetry the system has twisted, as well as untwisted, sectors. In this paper we consider the $Z_{2}$ orbifold model, where the twist operation is restricted to $Z_{2}$, which is a finite Abelian subgroup of $U(1)$. The two fields $\eta$ and $\xi$ are conveniently combined into a two-component symplectic fermion [6, [7] of conformal dimension 1 as

$$
\chi^{+}=\eta, \quad \chi^{-}=\partial \xi .
$$

This $Z_{2}$ orbifold symplectic fermion differs from the $\eta-\xi$ system (11) in the treatment of the $\xi$ zero mode, and its 
bosonic sector has been shown to be identical to the socalled triplet model [7], which is one of the few models of LCFT that have been studied extensively. Mode operators are defined by $\chi^{ \pm}(z)=\sum_{m \in Z+\lambda} \chi_{m}^{ \pm} z^{-m-1}$ and $\bar{\chi}^{ \pm}(\bar{z})=\sum_{m \in Z+\lambda} \bar{\chi}_{m}^{ \pm} \bar{z}^{-m-1}$, where $\lambda=0$ for the untwisted (Neveu-Schwartz) sector and $\lambda=1 / 2$ for the $Z_{2}$-twisted (Ramond) sector. Anticommutation relations consistent with the operator product of $\eta$ and $\xi$ are

$$
\left\{\chi_{m}^{\alpha}, \chi_{n}^{\beta}\right\}=m d^{\alpha \beta} \delta_{m+n},
$$

where $d^{\alpha \beta}$ is an antisymmetric tensor such that $d^{ \pm \mp}=$ \pm 1 , and $d_{\alpha \beta}$ is defined by $d^{\alpha \gamma} d_{\gamma \beta}=\delta_{\beta}^{\alpha}$. The normal ordering in the twisted sector is defined on the twisted vacua, and the formulae are obtained using the operator products of the symplectic fermions with twist operators [1].7.8, or resorting to the twisted Borcherds identity [9,10] in operator formalism. Then the energymomentum tensor in terms of the generalized normal ordering becomes $T(z)=d_{\alpha \beta}: \chi^{\alpha}(z) \chi^{\beta}(z): / 2+\lambda(\lambda-$ $1) / 2$, and from this, the Virasoro operators are given as

$$
L_{n}=\frac{1}{2} d_{\alpha \beta} \sum_{m \in Z+\lambda}: \chi_{m}^{\alpha} \chi_{n-m}^{\beta}:+\frac{\lambda(\lambda-1)}{2} \delta_{n 0} .
$$

In the twisted sector, the ground state is unique and is denoted by $\mu$. This has weight $-1 / 8$ and is identified as the ground state of the representation $\mathcal{V}_{-1 / 8}$. There is another representation $\mathcal{V}_{3 / 8}$ in this sector, which is built on the state $\nu^{\alpha}=\chi_{-1 / 2}^{\alpha} \mu$, with weight $3 / 8$. The untwisted sector is more complicated because the existence of a zero-mode leads to quadruply degenerate ground states. Let $\omega$ be a state annihilated by $\chi_{m>0}^{\alpha}$. Then, the four degenerate ground states are $\omega, \theta^{ \pm}=-\chi_{0}^{ \pm} \omega$ and $\Omega=\chi_{0}^{-} \chi_{0}^{+} \omega=L_{0} \omega$. Note that $\Omega$ is annihilated by further operations of zero-modes, and hence identified as the Möbius invariant vacuum. The bosonic ground states $\Omega$ and $\omega$ span a two-dimensional Jordan cell on the action of $L_{0}$, and they are said to form a logarithmic pair. Then $\Omega$ and $\omega$ constitute an irreducible vacuum representation $\mathcal{V}_{0}$, which is a sub-representation of the "reducible but indecomposable [11, 12]" representation $\mathcal{R}_{0}$. There is another such logarithmic pair, $\phi^{\alpha}=\chi_{-1}^{\alpha} \omega$ and $\psi^{\alpha}=\chi_{-1}^{\alpha} \Omega$, constituting an irreducible representation $\mathcal{V}_{1}$, which is again included in a reducible but indecomposable larger representation $\mathcal{R}_{1}$. These six representations, $\mathcal{V}_{-1 / 8}, \mathcal{V}_{3 / 8}, \mathcal{V}_{0}, \mathcal{V}_{1}, \mathcal{R}_{0}$ and $\mathcal{R}_{1}$, are the building blocks of the $Z_{2}$ orbifold $c=-2$ model. We emphasize that these six are not all independent since $\mathcal{V}_{0}$ and $\mathcal{V}_{1}$ are sub-representations of $\mathcal{R}_{0}$ and $\mathcal{R}_{1}$, respectively. The characters for these representations are calculated in 6, 7, 13, as:

$$
\begin{aligned}
& \chi_{\mathcal{R}_{0}}(\tau)=\chi_{\mathcal{R}_{1}}(\tau)=2 \Lambda_{1,2}(\tau), \\
& \chi \mathcal{V}_{0}(\tau)=\frac{1}{2} \Lambda_{1,2}(\tau)+\frac{1}{2} \eta(\tau)^{2},
\end{aligned}
$$

$$
\begin{aligned}
& \chi_{\mathcal{V}_{1}}(\tau)=\frac{1}{2} \Lambda_{1,2}(\tau)-\frac{1}{2} \eta(\tau)^{2}, \\
& \chi_{\mathcal{V}_{-1 / 8}}(\tau)=\Lambda_{0,2}(\tau), \\
& \chi_{\mathcal{V}_{3 / 8}}(\tau)=\Lambda_{2,2}(\tau),
\end{aligned}
$$

where $\Lambda_{m n}(\tau)=\Theta_{m n}(\tau) / \eta(\tau)$. We define the modular parameter as $q=e^{2 \pi i \tau}$, and denote the Jacobi $\Theta$-function and Dedekind $\eta$-function by $\Theta_{m n}(\tau)=$ $\sum_{k \in Z} q^{(2 n k+m)^{2} / 4 n}$ and $\eta(\tau)=q^{1 / 24} \prod_{k \in Z_{+}}\left(1-q^{k}\right)$, respectively. The characters (5) do not close under the modular transformation $\tau \rightarrow \tilde{\tau}=-1 / \tau$ since $\eta(\tau)^{2}$ is transformed into $-i \tilde{\tau} \eta(\tilde{\tau})^{2}$. The fusion rules are proposed in 11, 14, as

$$
\begin{aligned}
\mathcal{R}_{i} \times \mathcal{R}_{j} & =2 \mathcal{R}_{0}+2 \mathcal{R}_{1} & & i, j=0,1 \\
\mathcal{R}_{i} \times \mathcal{V}_{j} & =\mathcal{R}_{0} & & (i, j)=(0,0),(1,1), \\
& =\mathcal{R}_{1} & & (i, j)=(0,1),(1,0), \\
& =2 \mathcal{V}_{-1 / 8}+2 \mathcal{V}_{3 / 8} & & i=0,1 ; j=-\frac{1}{8}, \frac{3}{8}, \\
\mathcal{V}_{i} \times \mathcal{V}_{j} & =\mathcal{V}_{0} & & (i, j)=(0,0),(1,1), \\
& =\mathcal{V}_{1} & & (i, j)=(0,1),(1,0) \\
& =\mathcal{V}_{-1 / 8} & & (i, j)=\left(0,-\frac{1}{8}\right),\left(1, \frac{3}{8}\right), \\
& =\mathcal{V}_{3 / 8} & & (i, j)=\left(1,-\frac{1}{8}\right),\left(0, \frac{3}{8}\right), \\
& =\mathcal{R}_{0} & & (i, j)=\left(-\frac{1}{8},-\frac{1}{8}\right),\left(\frac{3}{8}, \frac{3}{8}\right), \\
& =\mathcal{R}_{1} & & (i, j)=\left(-\frac{1}{8}, \frac{3}{8}\right),\left(\frac{3}{8},-\frac{1}{8}\right) .
\end{aligned}
$$

Since the four representations $\mathcal{R}_{0}, \mathcal{R}_{1}, \mathcal{V}_{-1 / 8}$ and $\mathcal{V}_{3 / 8}$ close under the fusion, this model is considered as a rational conformal field theory, with a slightly weakened definition of rationality [1].

\section{BOUNDARY STATES OF THE SYMPLECTIC FERMION}

Now let us construct boundary states from the action (11). Consider a cylinder of circumference $L$ and length $T$. Modular parameters are defined as $\tilde{q}=e^{-4 \pi T / L}$ and $\tilde{\tau}=2 i T / L$. As in the construction of Dirichlet and Neumann boundary states in open superstring theory [15 17], we assume that the boundary term in the action vanishes, $\eta \xi+\bar{\eta} \bar{\xi}=0$. Since $\eta$ and $\xi$ have different scaling dimensions, this condition is decomposed into the linear conditions that $\eta=e^{i \phi} \bar{\eta}$ and $\xi=e^{i(\pi-\phi)} \bar{\xi}$ on the boundary. Here, $\phi$ is a phase factor reflecting the $U(1)$ symmetry of the system. These conditions are trivially consistent with the conformal invariance. Although it might be possible to include non-trivial interactions between bulk and boundary by introducing conformally invariant boundary terms, we do not consider such terms here. On Fourier decomposition of the symplectic fermion into mode operators, this condition is rephrased as constraints on boundary states $|B\rangle$ and $\langle B|$ at boundaries as

$$
\begin{aligned}
& \left(\chi_{m}^{ \pm}-e^{ \pm i \phi} \bar{\chi}_{-m}^{ \pm}\right)|B\rangle=0, \\
& \langle B|\left(\chi_{m}^{ \pm}-e^{ \pm i \phi} \bar{\chi}_{-m}^{ \pm}\right)=0 .
\end{aligned}
$$


These equations are satisfied by coherent states,

$$
\begin{aligned}
& \left|B_{0 \phi}\right\rangle=N \exp \left(\sum_{k>0} \frac{e^{i \phi}}{k} \chi_{-k}^{-} \bar{\chi}_{-k}^{+}+\frac{e^{-i \phi}}{k} \bar{\chi}_{-k}^{-} \chi_{-k}^{+}\right)\left|0_{\phi}\right\rangle \\
& \left\langle B_{0 \phi}\right|=\left\langle 0_{\phi}\right| N^{*} \exp \left(\sum_{k>0} \frac{e^{i \phi}}{k} \chi_{k}^{-} \bar{\chi}_{k}^{+}+\frac{e^{-i \phi}}{k} \bar{\chi}_{k}^{-} \chi_{k}^{+}\right),
\end{aligned}
$$

where $N$ and $N^{*}$ are normalization constants, and $k$ runs over integers in the untwisted sector and half-integers in the twisted sector. $\left|0_{\phi}\right\rangle$ stands for a non-chiral ground state satisfying

$$
\chi_{k>0}^{ \pm}\left|0_{\phi}\right\rangle=0=\bar{\chi}_{k>0}^{ \pm}\left|0_{\phi}\right\rangle .
$$

In addition to this, $\left|0_{\phi}\right\rangle$ in the untwisted sector must satisfy

$$
\left(\chi_{0}^{ \pm}-e^{ \pm i \phi} \bar{\chi}_{0}^{ \pm}\right)\left|0_{\phi}\right\rangle=0
$$

The bra-ground state $\left\langle 0_{\phi}\right|$ satisfies similar conditions.

The non-chiral ground state $\left|0_{\phi}\right\rangle$ in the twisted sector is merely a direct product $|\mu \otimes \bar{\mu}\rangle$. In the untwisted sector, there are two chiral bosonic ground states $\omega$ and $\Omega$, and therefore we have four non-chiral ground states, namely, $|\omega \otimes \bar{\omega}\rangle,|\omega \otimes \bar{\Omega}\rangle,|\Omega \otimes \bar{\omega}\rangle$ and $|\Omega \otimes \bar{\Omega}\rangle$. Among these, $|\omega \otimes \bar{\Omega}\rangle$ and $|\Omega \otimes \bar{\omega}\rangle$ are not consistent with $(10)$ since $\chi_{0}^{ \pm} \omega \neq 0$ and $\chi_{0}^{ \pm} \Omega=0$. The condition (10) non-trivially connects the left and right sectors of $|\omega \otimes \bar{\omega}\rangle$ through the phase factor $\phi$, whereas $|\Omega \otimes \bar{\Omega}\rangle$ trivially satisfies $(10)$. Thus we have three non-chiral ground states $|\omega \otimes \bar{\omega}\rangle,|\Omega \otimes \bar{\Omega}\rangle$, and $|\mu \otimes \bar{\mu}\rangle$. We normalize these states to be

$$
\begin{aligned}
& \langle\omega \otimes \bar{\omega} \mid \omega \otimes \bar{\omega}\rangle=\kappa, \\
& \langle\Omega \otimes \bar{\Omega} \mid \omega \otimes \bar{\omega}\rangle=\langle\omega \otimes \bar{\omega} \mid \Omega \otimes \bar{\Omega}\rangle=\rho \equiv-1, \\
& \langle\mu \otimes \bar{\mu} \mid \mu \otimes \bar{\mu}\rangle=1 .
\end{aligned}
$$

For the convenience of later discussions we have chosen $\rho=-1$. We leave the value of $\kappa$ unfixed. The boundary states (8) are indexed by the phase factor $\phi$ and the choice of ground state $0_{\phi}$ which may also depend on $\phi$. With a slight misuse of the notation we shall denote in the sequel $\omega \otimes \bar{\omega}, \Omega \otimes \bar{\Omega}$ and $\mu \otimes \bar{\mu}$ as $\omega, \Omega$ and $\mu$, respectively.

The condition of continuity for a general chiral field $J$ across the boundary $\zeta=\bar{\zeta}$ is

$$
[J(\zeta)-\bar{J}(\bar{\zeta})]_{\zeta=\bar{\zeta}}=0
$$

A semi-annular domain in the upper half $\zeta$-plane is conformally mapped onto a full annulus in the $z$-plane by $z=\exp (-2 \pi i w / L)$ and $w=(T / \pi) \ln \zeta$. Under this mapping the boundary $\zeta=\bar{\zeta}$ is mapped to $|z|=1$, $\exp (2 \pi T / L)$, and the condition (12) reads

$$
z^{s_{J}} J(z)=(-\bar{z})^{s_{J}} \bar{J}(\bar{z})
$$

on the boundary. Here, $s_{J}$ is the spin of $J$. Now that the $z$-plane allows radial quantization, the continuity of $J$ may be translated into conditions on the boundary states as 4,18

$$
\left(J_{m}-(-1)^{s_{J}} \bar{J}_{-m}\right)\left|B_{0 \phi}\right\rangle=0 .
$$

When $J(z)$ is the energy-momentum tensor $T(z)$, (14) reduces to the diffeomorphism invariance of the boundary

$$
\left(L_{m}-\bar{L}_{-m}\right)\left|B_{0 \phi}\right\rangle
$$

Recalling that the Virasoro operators are given as (4), it is easily verified that indeed the states (8) satisfy this condition. There are extra symmetries in our system other than the conformal invariance, as is hinted by the $U(1)$ invariance of the action (1). In fact, the symmetry is upgraded to $S U(2)$ through the re-formulation into the symplectic fermion. Combining the $s u(2)$ and the Virasoro algebra, our system has a W-algebra of type $\mathcal{W}\left(2,3^{3}\right)$ 6, 10,11, 19, which is generated by the Virasoro operators (of weight 2) plus three operators of weight 3 :

$$
\begin{aligned}
& W^{0}=-\frac{1}{2}\left\{: \partial \chi^{+} \chi^{-}:+: \partial \chi^{-} \chi^{+}:\right\}, \\
& W^{ \pm}=: \partial \chi^{ \pm} \chi^{ \pm}:
\end{aligned}
$$

where the normal ordering is taken on appropriate (twisted or untwisted) vacuum for each sector. The mode operators $W_{m}^{a}$ are

$$
\begin{aligned}
W_{n}^{0} & =-\frac{1}{2} \sum_{j \in Z+\lambda} j\left\{: \chi_{n-j}^{+} \chi_{j}^{-}:+: \chi_{n-j}^{-} \chi_{j}^{+}:\right\}, \\
W_{n}^{ \pm} & =\sum_{j \in Z+\lambda} j \chi_{n-j}^{ \pm} \chi_{j}^{ \pm}
\end{aligned}
$$

where $\lambda=0$ for the untwisted and $1 / 2$ for the twisted sector as before. Since the $\mathrm{W}$-operators have spin 3 , the continuity condition for the $\mathrm{W}$-field reads

$$
\left(W_{m}^{a}+\bar{W}_{-m}^{a}\right)\left|B_{0 \phi}\right\rangle=0 .
$$

Besides (10) which has already been imposed for the conformal invariance, (18) implies an extra condition

$$
e^{2 i \phi}=1
$$

which means that the values of $\phi$ must be 0 modulo $\pi$. Thus in our $Z_{2}$ orbifold model, we restrict the values of $\phi$ to be either $\phi=0$ or $\phi=\pi$, and in the following text we write $\left|B_{0+}\right\rangle=\left|B_{0, \phi=0}\right\rangle,\left|B_{0-}\right\rangle=\left|B_{0, \phi=\pi}\right\rangle$, and $\left\langle B_{0+}\right|=\left\langle B_{0, \phi=0}\right|,\left\langle B_{0-}\right|=\left\langle B_{0, \phi=\pi}\right|$. Then we have six distinct boundary states, $\left|B_{\omega+}\right\rangle,\left|B_{\omega-}\right\rangle,\left|B_{\Omega+}\right\rangle$, $\left|B_{\Omega-}\right\rangle,\left|B_{\mu+}\right\rangle$ and $\left|B_{\mu-}\right\rangle$, which we collectively write $|a\rangle=\left|B_{0 \phi}\right\rangle$. Once boundary states are constructed, the boundary-to-boundary amplitudes (partition functions on the cylinder $\left\langle a\left|\left(\tilde{q}^{1 / 2}\right)^{L_{0}+\bar{L}_{0}+1 / 6}\right| b\right\rangle$ are calculated. Setting $|N|^{2}=1$, they are summarized in Table 1. 
Strictly speaking, the boundary states (8) are not normalizable. Their non-trivial inner products are

$$
\begin{aligned}
& \left\langle B_{\omega \pm} \mid B_{\omega \mp}\right\rangle=\kappa \lim _{\tilde{\tau} \rightarrow 0} \Lambda_{1,2}(\tilde{\tau}), \\
& \left\langle B_{\Omega \pm} \mid B_{\omega \mp}\right\rangle=\left\langle B_{\omega \pm} \mid B_{\Omega \mp}\right\rangle=-\lim _{\tilde{\tau} \rightarrow 0} \Lambda_{1,2}(\tilde{\tau}), \\
& \left\langle B_{\mu \pm} \mid B_{\mu \mp}\right\rangle=\lim _{\tilde{\tau} \rightarrow 0}\left(\Lambda_{0,2}(\tilde{\tau})+\Lambda_{2,2}(\tilde{\tau})\right) .
\end{aligned}
$$

The right hand sides of $(20)$ are all divergent, which is a well-known feature of such boundary states.

\section{MODULAR PROPERTIES OF THE BOUNDARY STATES}

The amplitude $\left\langle a\left|\left(\tilde{q}^{1 / 2}\right)^{L_{0}+\bar{L}_{0}+1 / 6}\right| b\right\rangle$ represents the tree-level graph of a closed string propagating from one boundary to the other. The same amplitude describes the one-loop graph of an open string having specific boundary states at each end. This equivalence imposes strong constraints on the physical boundary states. Assume an open string has boundary states $\tilde{\alpha}$ and $\tilde{\beta}$ at its ends. If these boundary states are physical, $n_{\tilde{\alpha} \tilde{\beta}}^{i}$ (a nonnegative integer) copies of representations $i$ appear in the bulk. The partition function is then the sum of the bulk characters and written as $Z_{\tilde{\alpha} \tilde{\beta}}(q)=\sum_{i} n_{\tilde{\alpha} \tilde{\beta}}^{i} \chi_{i}(q)$, where $\chi_{i}(q)$ are the characters for representations $i$, and $q=e^{-\pi L / T}$. Duality of the open and closed channels demands $Z_{\tilde{\alpha} \tilde{\beta}}(q)=\left\langle\tilde{\alpha}\left|\left(\tilde{q}^{1 / 2}\right)^{L_{0}+\bar{L}_{0}+1 / 6}\right| \tilde{\beta}\right\rangle$. In the literature this is often called Cardy's relation [4]. On expanding physical boundary states by the boundary states we constructed above, we obtain

$$
\sum_{i} n_{\tilde{\alpha} \tilde{\beta}}^{i} \chi_{i}(q)=\sum_{a, b}\langle\tilde{\alpha} \mid a\rangle\left\langle a\left|\left(\tilde{q}^{1 / 2}\right)^{L_{0}+\bar{L}_{0}+1 / 6}\right| b\right\rangle\langle b \mid \tilde{\beta}\rangle .
$$

Here, $\langle\tilde{\alpha} \mid a\rangle$ and $\langle b \mid \tilde{\beta}\rangle$ should be understood merely as coefficients in expansions $\langle\tilde{\alpha}|=\sum_{a}\langle\tilde{\alpha} \mid a\rangle\langle a|$, and $|\tilde{\beta}\rangle=$ $\sum_{b}\langle b \mid \tilde{\beta}\rangle|b\rangle$, as ordinary orthonormal bra-ket operations are not possible in our case.

Using the modular transformations of Jacobi and Dedekind functions [20], relations between $|a\rangle$ and $|\tilde{\alpha}\rangle$ are found by comparing the coefficients of functions on both sides. Some care is needed: all non-diagonal contributions have to be considered since on the right hand side amplitudes are not diagonalized into characters. Equating the coefficients of $\Lambda_{1,2}(\tilde{\tau}) \ln \tilde{q}, \eta(\tilde{\tau})^{2} \ln \tilde{q}, \Lambda_{1,2}(\tilde{\tau})$, $\eta(\tilde{\tau})^{2}, \Lambda_{0,2}(\tilde{\tau})+\Lambda_{2,2}(\tilde{\tau})$ and $\Lambda_{0,2}(\tilde{\tau})-\Lambda_{2,2}(\tilde{\tau})$, we have

$$
\begin{aligned}
& \left\langle\tilde{\alpha} \mid B_{\omega+}\right\rangle\left\langle B_{\omega-} \mid \tilde{\beta}\right\rangle+\left\langle\tilde{\alpha} \mid B_{\omega-}\right\rangle\left\langle B_{\omega+} \mid \tilde{\beta}\right\rangle=0, \\
& \left\langle\tilde{\alpha} \mid B_{\omega+}\right\rangle\left\langle B_{\omega+} \mid \tilde{\beta}\right\rangle+\left\langle\tilde{\alpha} \mid B_{\omega-}\right\rangle\left\langle B_{\omega-} \mid \tilde{\beta}\right\rangle=\frac{n_{\tilde{\alpha} \tilde{\beta}}^{\mathcal{V}_{0}}-n_{\tilde{\alpha} \tilde{\beta}}^{\mathcal{V}_{1}}}{4 \pi}, \quad(23) \\
& \left\langle\tilde{\alpha} \mid B_{\omega+}\right\rangle\left\langle B_{\Omega-} \mid \tilde{\beta}\right\rangle+\left\langle\tilde{\alpha} \mid B_{\omega-}\right\rangle\left\langle B_{\Omega+} \mid \tilde{\beta}\right\rangle+\left\langle\tilde{\alpha} \mid B_{\Omega+}\right\rangle\left\langle B_{\omega-} \mid \tilde{\beta}\right\rangle \\
& +\left\langle\tilde{\alpha} \mid B_{\Omega-}\right\rangle\left\langle B_{\omega+} \mid \tilde{\beta}\right\rangle=n_{\tilde{\alpha} \tilde{\beta}}^{\mathcal{V}_{3 / 8}}-n_{\tilde{\alpha} \tilde{\beta}}^{\mathcal{V}_{-1 / 8}},
\end{aligned}
$$

$$
\begin{aligned}
& \left\langle\tilde{\alpha} \mid B_{\omega+}\right\rangle\left\langle B_{\Omega+} \mid \tilde{\beta}\right\rangle+\left\langle\tilde{\alpha} \mid B_{\omega-}\right\rangle\left\langle B_{\Omega-} \mid \tilde{\beta}\right\rangle \\
& +\left\langle\tilde{\alpha} \mid B_{\Omega+}\right\rangle\left\langle B_{\omega+} \mid \tilde{\beta}\right\rangle+\left\langle\tilde{\alpha} \mid B_{\Omega-}\right\rangle\left\langle B_{\omega-} \mid \tilde{\beta}\right\rangle \\
& -\kappa\left(\left\langle\tilde{\alpha} \mid B_{\omega-}\right\rangle\left\langle B_{\omega-} \mid \tilde{\beta}\right\rangle+\left\langle\tilde{\alpha} \mid B_{\omega+}\right\rangle\left\langle B_{\omega+} \mid \tilde{\beta}\right\rangle\right)=0 \\
& \left\langle\tilde{\alpha} \mid B_{\mu+}\right\rangle\left\langle B_{\mu-} \mid \tilde{\beta}\right\rangle+\left\langle\tilde{\alpha} \mid B_{\mu-}\right\rangle\left\langle B_{\mu+} \mid \tilde{\beta}\right\rangle=\frac{n_{\tilde{\alpha} \tilde{\beta}}^{\mathcal{V}_{-1 / 8}}+n_{\tilde{\alpha} \tilde{\beta}}^{\mathcal{V}_{3 / 8}}}{2} \\
& \left\langle\tilde{\alpha} \mid B_{\mu+}\right\rangle\left\langle B_{\mu+} \mid \tilde{\beta}\right\rangle+\left\langle\tilde{\alpha} \mid B_{\mu-}\right\rangle\left\langle B_{\mu-} \mid \tilde{\beta}\right\rangle \\
& =n_{\tilde{\alpha} \tilde{\beta}}^{\mathcal{R}_{0}}+n_{\tilde{\alpha} \tilde{\beta}}^{\mathcal{R}_{1}}+\frac{1}{4}\left(n_{\tilde{\alpha} \tilde{\beta}}^{\mathcal{V}_{0}}+n_{\tilde{\alpha} \tilde{\beta}}^{\mathcal{V}_{1}}\right)
\end{aligned}
$$

Now following Cardy's fusion method [4], let us find the consistent physical boundary states one by one. We assume that bra and ket boundary states have the same real coefficients $\langle a \mid \tilde{\alpha}\rangle=\langle\tilde{\alpha} \mid a\rangle$. We start by looking for a reference state $\left|\tilde{\mathcal{V}}_{0}\right\rangle$ such that $n_{\tilde{\mathcal{V}}_{0} \tilde{\alpha}}^{i}=n_{\tilde{\alpha} \tilde{\mathcal{V}}_{0}}^{i}=\delta_{\tilde{\alpha}}^{i}$. Let $\tilde{\alpha}=\tilde{\beta}=\tilde{\mathcal{V}}_{0}$ in (22)-(27). In the untwisted sector, from (22) we have $\left\langle\tilde{\mathcal{V}}_{0} \mid B_{\omega+}\right\rangle\left\langle B_{\omega-} \mid \tilde{\mathcal{V}}_{0}\right\rangle=0$. Since we can exchange $\phi=0$ and $\phi=\pi$ as a consequence of $Z_{2}$ symmetry, we may put $\left\langle\tilde{\mathcal{V}}_{0} \mid B_{\omega+}\right\rangle=\left\langle B_{\omega+} \mid \tilde{\mathcal{V}}_{0}\right\rangle=0$ without loss of generality. Then from $(23)$ we have $\left|\left\langle\tilde{\mathcal{V}}_{0} \mid B_{\omega-}\right\rangle\right|^{2}=$ $1 /(4 \pi)$, so $\left\langle\tilde{\mathcal{V}}_{0} \mid B_{\omega-}\right\rangle=\left\langle B_{\omega-} \mid \mathcal{V}_{0}\right\rangle=1 /(2 \sqrt{\pi})$. Substituting these values, (24) gives $\left\langle\tilde{\mathcal{V}}_{0} \mid B_{\Omega+}\right\rangle=\left\langle B_{\Omega+} \mid \tilde{\mathcal{V}}_{0}\right\rangle=0$. From (25) we find $\left\langle\mathcal{V}_{0} \mid B_{\Omega-}\right\rangle=\left\langle B_{\Omega-} \mid \tilde{\mathcal{V}}_{0}\right\rangle=\kappa /(4 \sqrt{\pi})$. In the twisted sector, 26) becomes $\left\langle\tilde{\mathcal{V}}_{0} \mid B_{\mu+}\right\rangle\left\langle B_{\mu-} \mid \tilde{\mathcal{V}}_{0}\right\rangle=$ 0 , and again without losing generality we can choose $\left\langle\tilde{\mathcal{V}}_{0} \mid B_{\mu+}\right\rangle=\left\langle B_{\mu+} \mid \tilde{\mathcal{V}}_{0}\right\rangle=0$. Then from (27) we find $\left\langle\tilde{\mathcal{V}}_{0} \mid B_{\mu-}\right\rangle=\left\langle B_{\mu-} \mid \tilde{\mathcal{V}}_{0}\right\rangle=1 / 2$. Thus we found $\left|\tilde{\mathcal{V}}_{0}\right\rangle=$ $(1 / 2 \sqrt{\pi})\left|B_{\omega-}\right\rangle+(\kappa / 4 \sqrt{\pi})\left|B_{\Omega-}\right\rangle+(1 / 2)\left|B_{\mu-}\right\rangle$.

Next, we put $\tilde{\alpha}=\tilde{\mathcal{V}}_{1}$ and $\tilde{\beta}=\tilde{\mathcal{V}}_{0}$. We find $\left\langle\tilde{\mathcal{V}}_{1} \mid B_{\omega-}\right\rangle=\left\langle B_{\omega-} \mid \tilde{\mathcal{V}}_{1}\right\rangle=-1 /(2 \sqrt{\pi}),\left\langle\tilde{\mathcal{V}}_{1} \mid B_{\Omega-}\right\rangle=$ $\left\langle B_{\Omega-} \mid \tilde{\mathcal{V}}_{1}\right\rangle=-\kappa /(4 \sqrt{\pi}),\left\langle\tilde{\mathcal{V}}_{1} \mid B_{\mu-}\right\rangle=\left\langle B_{\mu-} \mid \tilde{\mathcal{V}}_{1}\right\rangle=1 / 2$, and $\left\langle\tilde{\mathcal{V}}_{1} \mid B_{\omega+}\right\rangle=\left\langle B_{\omega+} \mid \tilde{\mathcal{V}}_{1}\right\rangle=\left\langle\tilde{\mathcal{V}}_{1} \mid B_{\Omega+}\right\rangle=\left\langle B_{\Omega+} \mid \tilde{\mathcal{V}}_{1}\right\rangle=$ $\left\langle\tilde{\mathcal{V}}_{1} \mid B_{\mu+}\right\rangle=\left\langle B_{\mu+} \mid \tilde{\mathcal{V}}_{1}\right\rangle=0$. The rest of the states are found similarly by putting $\tilde{\alpha}=\tilde{\mathcal{V}}_{-1 / 8}, \tilde{\mathcal{V}}_{3 / 8}, \tilde{\mathcal{R}}_{0}, \tilde{\mathcal{R}}_{1}$ one by one, all with $\tilde{\beta}=\tilde{\mathcal{V}}_{0}$. Then we find

$$
\begin{aligned}
& \left|\tilde{\mathcal{V}}_{0}\right\rangle=\frac{1}{2 \sqrt{\pi}}\left|B_{\omega-}\right\rangle+\frac{\kappa}{4 \sqrt{\pi}}\left|B_{\Omega-}\right\rangle+\frac{1}{2}\left|B_{\mu-}\right\rangle, \\
& \left|\tilde{\mathcal{V}}_{1}\right\rangle=\frac{-1}{2 \sqrt{\pi}}\left|B_{\omega-}\right\rangle-\frac{\kappa}{4 \sqrt{\pi}}\left|B_{\Omega-}\right\rangle+\frac{1}{2}\left|B_{\mu-}\right\rangle, \\
& \left|\tilde{\mathcal{V}}_{-1 / 8}\right\rangle=\left|B_{\mu+}\right\rangle-2 \sqrt{\pi}\left|B_{\Omega+}\right\rangle, \\
& \left|\tilde{\mathcal{V}}_{3 / 8}\right\rangle=\left|B_{\mu+}\right\rangle+2 \sqrt{\pi}\left|B_{\Omega+}\right\rangle, \\
& |\tilde{\mathcal{R}}\rangle \equiv\left|\tilde{\mathcal{R}}_{0}\right\rangle=\left|\tilde{\mathcal{R}}_{1}\right\rangle=2\left|B_{\mu-}\right\rangle .
\end{aligned}
$$

Since $\tilde{\mathcal{R}}_{0}$ and $\tilde{\mathcal{R}}_{1}$ are the same state, we shall denote it as $\tilde{\mathcal{R}}$. There are other solutions obtained from the above by exchanging $\omega+$ and $\omega-, \Omega+$ and $\Omega-, \mu+$ and $\mu-$ (first and second pairs have to be exchanged simultaneously), as a consequence of the $Z_{2}$ symmetry. Apart from this, the solutions are unique. Therefore, the duality of open and closed string channels provides strong enough constraints for the physical boundary states to be determined without ambiguity. Substituting these states back 
into (21), possible $n_{\tilde{\alpha} \tilde{\beta}}^{i}$ on the left hand side are found. Note that $n_{\tilde{\alpha} \tilde{\beta}}^{i}$ cannot be determined uniquely by this procedure, since the characters are not independent but $\chi_{\mathcal{R}_{0}}=\chi_{\mathcal{R}_{1}}=2\left(\chi_{\mathcal{V}_{0}}+\chi_{\mathcal{V}_{1}}\right)$. Up to this ambiguity $n_{\tilde{\alpha} \tilde{\beta}}^{i}$ is identical to the fusion matrix $n_{j k}^{i}$, which appears as $\Phi_{j} \times \Phi_{k}=\sum_{i} n_{j k}^{i} \Phi_{i}$ ( $\Phi$ 's stand for the representations) in the fusion rule (6).

\section{DISCUSSION}

The essential point in our analysis is the appearance of the term $\eta(\tilde{\tau})^{2} \ln \tilde{q}$ in the cylinder amplitude (Table 1 ) through the proper treatment of the zero-mode. Note that the five modular functions $\eta(\tilde{\tau})^{2}, \eta(\tilde{\tau})^{2} \ln \tilde{q}, \Lambda_{0,2}(\tilde{\tau})$, $\Lambda_{1,2}(\tilde{\tau}), \Lambda_{2,2}(\tilde{\tau})$ close under the modular transformation $\tilde{\tau} \rightarrow-1 / \tilde{\tau}$. Discarding either $B_{\omega+}$ or $B_{\omega-}$ in order to get rid of the unwanted function $\Lambda_{1,2}(\tilde{\tau})^{2} \ln \tilde{q}$, we obtained a set of boundary states including the reference state $\tilde{\mathcal{V}}_{0}$ which is necessary for the Cardy fusion procedure. This situation is quite similar to what happens in the Ising model case 21 23], where one of the two $R$ sector states has to be discarded to give three boundary states, namely spin up, down, and free, which behave appropriately under modular transformations. In fact, the Ising model is the only known example which allows free-field construction of boundary states.

However, our model differs from the Ising model in one important respect. Neglecting the row and column involving the discarded state $B_{\omega+}$, the cylinder amplitude of the untwisted sector in Table 1 gives a matrix

$$
\left(\begin{array}{ccc}
(\kappa-\ln \tilde{q}) \eta(\tilde{\tau})^{2} & -\Lambda_{1,2}(\tilde{\tau}) & -\eta(\tilde{\tau})^{2} \\
-\Lambda_{1,2}(\tilde{\tau}) & 0 & 0 \\
-\eta(\tilde{\tau})^{2} & 0 & 0
\end{array}\right)
$$

which is not regular. Since one of the three eigen-values is zero, the untwisted sector has only two non-trivial partition functions on diagonalization. This means that the net content of the space spanned by $\left|B_{\omega-}\right\rangle,\left|B_{\Omega \pm}\right\rangle,\left|B_{\mu \pm}\right\rangle$ consists of only four states, not five. Therefore it is not possible to allocate five boundary states to the five modular functions.

This is related to the difficulty in expressing the physical boundary states in terms of the Ishibashi states. In ordinary CFTs, the solutions to (15) are found in the form of Ishibashi states [18]

$$
|j\rangle\rangle \equiv \sum_{M}|j ; M\rangle \otimes U \overline{|j ; M\rangle},
$$

where $U$ is an antiunitary operator, $j$ is a label for Verma modules, and $M$ is a level in the module. Ishibashi states diagonalize the cylinder amplitudes to give characters,

$$
\begin{aligned}
& \left\langle\left\langle i\left|\left(\tilde{q}^{1 / 2}\right)^{L_{0}+\bar{L}_{0}-c / 12}\right| i\right\rangle\right\rangle=\chi_{i}(\tilde{q}), \\
& \left\langle\left\langle i\left|\left(\tilde{q}^{1 / 2}\right)^{L_{0}+\bar{L}_{0}-c / 12}\right| j\right\rangle\right\rangle=0 \text { for } i \neq j .
\end{aligned}
$$

In our model, we can find candidates for the Ishibashi states such as

$$
\begin{aligned}
& \left.\left|\mathcal{V}_{0}\right\rangle\right\rangle=\frac{1}{2}\left|B_{\Omega+}\right\rangle+\frac{1}{2}\left|B_{\Omega-}\right\rangle, \\
& \left.\left|\mathcal{V}_{1}\right\rangle\right\rangle=\frac{1}{2}\left|B_{\Omega+}\right\rangle-\frac{1}{2}\left|B_{\Omega-}\right\rangle, \\
& \left.\left|\mathcal{V}_{-1 / 8}\right\rangle\right\rangle=\frac{1}{2}\left|B_{\mu+}\right\rangle+\frac{1}{2}\left|B_{\mu-}\right\rangle, \\
& \left.\left|\mathcal{V}_{3 / 8}\right\rangle\right\rangle=\frac{1}{2}\left|B_{\mu+}\right\rangle-\frac{1}{2}\left|B_{\mu-}\right\rangle, \\
& \left.\left.|\mathcal{R}\rangle\rangle \equiv\left|\mathcal{R}_{0}\right\rangle\right\rangle=\left|\mathcal{R}_{1}\right\rangle\right\rangle=\sqrt{2}\left|B_{\Omega+}\right\rangle,
\end{aligned}
$$

and

$$
\begin{aligned}
& \left\langle\left\langle\mathcal{V}_{0}\right|=\frac{-1}{2}\left\langle B_{\omega-}\right|-\frac{1}{2}\left\langle B_{\omega+}\right|,\right. \\
& \left\langle\left\langle\mathcal{V}_{1}\right|=\frac{1}{2}\left\langle B_{\omega+}\right|-\frac{1}{2}\left\langle B_{\omega-}\right|,\right. \\
& \left\langle\left\langle\mathcal{V}_{-1 / 8}\right|=\frac{1}{2}\left\langle B_{\mu+}\right|+\frac{1}{2}\left\langle B_{\mu-}\right|\right. \\
& \left\langle\left\langle\mathcal{V}_{3 / 8}\right|=\frac{1}{2}\left\langle B_{\mu-}\right|-\frac{1}{2}\left\langle B_{\mu+}\right|,\right. \\
& \left\langle\langle \mathcal { R } | \equiv \left\langle\left\langle\mathcal{R}_{0}\right|=\left\langle\left\langle\mathcal{R}_{1}\right|=-\sqrt{2}\left\langle B_{\omega-}\right|\right.\right.\right.
\end{aligned}
$$

whereby the characters (5) are reproduced in the form (31), and the orthogonality (32) holds for $\mathcal{V}_{0}, \mathcal{V}_{1}, \mathcal{V}_{-1 / 8}$, and $\mathcal{V}_{3 / 8}$. Note that it is not possible to find such states with the same bra and ket coefficients. It can be easily checked that the physical boundary states $\tilde{\mathcal{V}}_{0}$ and $\tilde{\mathcal{V}}_{1}$ cannot be expressed as linear combinations of the states (33), (34). As a consequence, it is not possible to derive the Verlinde formula using the modular transformations as in [4], since $\tilde{\mathcal{V}}_{0}$ plays an essential role in such discussions. The failure of the Verlinde formula is indeed consistent with the fusion rule (6), which cannot be diagonalised.

Alternatively, the four representations $\mathcal{R}_{0}, \mathcal{R}_{1}, \mathcal{V}_{-1 / 8}$ and $\mathcal{V}_{3 / 8}$ can be regarded as fundamental constituents of the theory, since they themselves close under the fusion. It is argued by Kausch and Gaberdiel [24] that local and non-chiral bulk theory with finite multiplicity is given by three non-chiral representations, namely, $\mathcal{V}_{-1 / 8} \otimes \overline{\mathcal{V}}_{-1 / 8}$, $\mathcal{V}_{3 / 8} \otimes \overline{\mathcal{V}}_{3 / 8}$, and $\mathcal{R}$ which is a combination of $\left(\mathcal{R}_{0} \otimes\right.$ $\left.\overline{\mathcal{R}}_{0}\right) / \mathcal{N}_{0 \overline{0}}$ and $\left(\mathcal{R}_{1} \otimes \overline{\mathcal{R}}_{1}\right) / \mathcal{N}_{1 \overline{1}}$, where $\mathcal{N}_{0 \overline{0}}$ and $\mathcal{N}_{1 \overline{1}}$ are subspaces to be quotiented out. This is analogous to our result that the physical boundary states for $\mathcal{R}_{0}$ and $\mathcal{R}_{1}$ are identical. Considering the four representations $\mathcal{R}_{0}$, $\mathcal{R}_{1}, \mathcal{V}_{-1 / 8}$ and $\mathcal{V}_{3 / 8}$, we see from (28) and (33) that the physical ket-states and Ishibashi ket-states are related as

$$
\begin{aligned}
& \left.\left.|\tilde{\mathcal{R}}\rangle=2\left|\mathcal{V}_{-1 / 8}\right\rangle\right\rangle-2\left|\mathcal{V}_{3 / 8}\right\rangle\right\rangle, \\
& \left.\left.\left.\left|\tilde{\mathcal{V}}_{-1 / 8}\right\rangle=\left|\mathcal{V}_{-1 / 8}\right\rangle\right\rangle+\left|\mathcal{V}_{3 / 8}\right\rangle\right\rangle-\sqrt{2 \pi}|\mathcal{R}\rangle\right\rangle, \\
& \left.\left.\left.\left|\tilde{\mathcal{V}}_{3 / 8}\right\rangle=\left|\mathcal{V}_{-1 / 8}\right\rangle\right\rangle+\left|\mathcal{V}_{3 / 8}\right\rangle\right\rangle+\sqrt{2 \pi}|\mathcal{R}\rangle\right\rangle
\end{aligned}
$$

These are the combinations of $\left|B_{\mu \pm}\right\rangle$ and $\left|B_{\Omega+}\right\rangle$. However, the boundary bra-states for these representations cannot be expressed in terms of the corresponding 
Ishibashi bra-states (34), since the former are the combinations of $\left\langle B_{\mu \pm}\right|$ and $\left\langle B_{\Omega+}\right|$, whereas the latter are of $\left\langle B_{\mu \pm}\right|$ and $\left\langle B_{\omega-}\right|$. The candidate of the Ishibashi states (33), (34) are not unique, and alternatively, we can define such states so that the bra-states are linearly related to the physical boundary states, but then the ket-states cannot be. That is, it is possible to express the physical boundary states in terms of such Ishibashi states on either of the two boundaries, but not on both.

We started from the Lagrangian representation of the $c=-2$ LCFT model and presented a possible solution for physical boundary states. Modular invariance imposes tight enough constraints on the partition function to identify the boundary states which allow the appearance of bulk representations. Although we could find five possible physically consistent boundary states $\tilde{\mathcal{R}}, \tilde{\mathcal{V}}_{0}$, $\tilde{\mathcal{V}}_{1}, \tilde{\mathcal{V}}_{-1 / 8}$ and $\tilde{\mathcal{V}}_{3 / 8}$, their implication is still not evident. The three states $\tilde{\mathcal{R}}, \tilde{\mathcal{V}}_{-1 / 8}, \tilde{\mathcal{V}}_{3 / 8}$ may be considered as genuinely physical as they correspond to non-chiral bulk representations. However, this speculation is not necessarily persuasive. Among well-studied unitary minimal models, the 3 -state Potts model is known to possess a $\mathrm{W}$-algebra, and its complete boundary states were found quite recently 25 27. In that model, only the fixed and mixed boundary states are obtained by Cardy's method from the $\mathrm{W}$-invariant conformal towers; in order to obtain the complete set including "free" and "new" boundary states, all chiral representations from the Kac table not constrained by the W-symmetry had to be considered. Then in our $c=-2$ model, the boundary states $\tilde{\mathcal{V}}_{0}$ and $\tilde{\mathcal{V}}_{1}$ may well represent some boundary conditions of a statistical model since the $c_{2,1}$ Kac table indicates representations with conformal weights $0,1,-1 / 8$ and $3 / 8$. Further study of the properties of the states we have obtained requires analysis of particular statistical models, such as critical polymers [1]28]. These topics are beyond the scope of this paper.

\section{ACKNOWLEDGMENTS}

The authors appreciate stimulating discussions with I. I. Kogan and Y. Ishimoto.

[1] H. Saleur, Nucl. Phys. B382 (1992) 486.

[2] V. Gurarie, Nucl. Phys. B410 (1993)535.

[3] J. Cardy, Logarithmic correlations in quenched random magnets and polymers, cond-mat/9911024.

[4] J. Cardy, Nucl. Phys. B324 (1989) 581.

[5] I. I. Kogan and J. F. Wheater, Phys.Lett. B486 (2000) 353 , for revised version see hep-th/0003184.

[6] H. G. Kausch, Curiosities at $c=-2$, hep-th/9510149.
[7] H. G. Kausch, Nucl.Phys. B583 (2000) 513.

[8] L. Dixon, D, Friedan, E. Martinec and S. Shenker, Nucl. Phys. B282 (1987) 13.

[9] V. Kac and A. Radul, Representation theory of the vertex algebra $W_{1+\infty}$, hep-th/9512150.

[10] W. Eholzer, L. Fehér and A. Honecker, Nucl. Phys. B518 (1998) 669.

[11] M. R. Gaberdiel and H. G. Kausch, Phys. Lett. B386 (1996) 131.

[12] F. Rohsiepe, On reducible but indecomposable representations of the Virasoro algebra, hep-th/9611160.

[13] M. A. I. Flohr, Int. J. Mod. Phys., A11 (1996) 4147; ibid. A12 (1997) 1943.

[14] M. R. Gaberdiel and H. G. Kausch, Nucl. Phys. B477 (1996) 293.

[15] C. G. Callan, C. Lovelace, C. R. Nappi and S. A. Yost, Nucl. Phys. B293 (1987) 83.

[16] J. Polchinski and Y. Cai, Nucl. Phys. B296 (1988) 91.

[17] N. Ishibashi and T. Onogi, Nucl. Phys. B318 (1989) 239.

[18] N. Ishibashi, Mod. Phys. Lett. A4 (1989) 251.

[19] H. G. Kausch, Phys. Lett. B259 (1991) 448.

[20] See e.g. A. Erdélyi, W. Magnus, F. Oberhettinger and F. G. Tricomi, Higher transcendental functions, vol. 2, McGraw-Hill, New York (1953).

[21] A. LeClair, G. Mussardo, H. Saleur and S. Skorik, Nucl. Phys. B453 (1995) 581.

[22] S. Yamaguchi, Prog. Theor. Phys. 97 (1997) 703.

[23] R. I. Nepomechie, Consistent superconformal boundary states, hep-th/0102010.

[24] M. R. Gaberdiel and H. G. Kausch, Nucl. Phys. B538 (1999) 631.

[25] I. Affleck, M. Oshikawa and H. Saleur, J. Phys. A31 (1998) 5827.

[26] J. Fuchs and C. Schweigert, Nucl. Phys. B441 (1998) 141.

[27] R. E. Behrend, P. A. Pearce and J.-B. Zuber, Nucl. Phys. A31 (1998) L763.

[28] B. Duplantier and H. Saleur, Nucl. Phys. B290 (1987) 291. 
Table 1: Amplitudes $\left\langle a\left|\left(\tilde{q}^{1 / 2}\right)^{L_{0}+\bar{L}_{0}+1 / 6}\right| b\right\rangle$.

Untwisted Sector

\begin{tabular}{c|c|c|c|c}
\hline \multirow{2}{*}{$\langle a|$} & \multicolumn{4}{|c}{$|c|$} \\
\cline { 2 - 5 } & $B_{\omega+}$ & $B_{\omega-}$ & $B_{\Omega+}$ & $B_{\Omega-}$ \\
\hline \hline$B_{\omega+}$ & $(\kappa-\ln \tilde{q}) \eta(\tilde{\tau})^{2}$ & $(\kappa-\ln \tilde{q}) \Lambda_{1,2}(\tilde{\tau})$ & $-\eta(\tilde{\tau})^{2}$ & $-\Lambda_{1,2}(\tilde{\tau})$ \\
\hline$B_{\omega-}$ & $(\kappa-\ln \tilde{q}) \Lambda_{1,2}(\tilde{\tau})$ & $(\kappa-\ln \tilde{q}) \eta(\tilde{\tau})^{2}$ & $-\Lambda_{1,2}(\tilde{\tau})$ & $-\eta(\tilde{\tau})^{2}$ \\
\hline$B_{\Omega+}$ & $-\eta(\tilde{\tau})^{2}$ & $-\Lambda_{1,2}(\tilde{\tau})$ & 0 & 0 \\
\hline$B_{\Omega-}$ & $-\Lambda_{1,2}(\tilde{\tau})$ & $-\eta(\tilde{\tau})^{2}$ & 0 & 0 \\
\hline
\end{tabular}

Twisted Sector

\begin{tabular}{c|c|c}
\hline \multirow{2}{*}{$\langle a|$} & \multicolumn{2}{|c}{$|b\rangle$} \\
\cline { 2 - 3 } & $B_{\mu+}$ & $B_{\mu-}$ \\
\hline \hline$B_{\mu+}$ & $\Lambda_{0,2}(\tilde{\tau})-\Lambda_{2,2}(\tilde{\tau})$ & $\Lambda_{0,2}(\tilde{\tau})+\Lambda_{2,2}(\tilde{\tau})$ \\
\hline$B_{\mu-}$ & $\Lambda_{0,2}(\tilde{\tau})+\Lambda_{2,2}(\tilde{\tau})$ & $\Lambda_{0,2}(\tilde{\tau})-\Lambda_{2,2}(\tilde{\tau})$ \\
\hline
\end{tabular}

